\title{
Macroscopic amplification of electroweak effects in molecular Bose-Einstein condensates
}

\author{
P. Bargueño and F. Sols \\ Departamento de Física de Materiales, Universidad Complutense de Madrid, E-28040, Madrid, Spain
}

\begin{abstract}
We investigate the possible use of Bose-Einstein condensates of diatomic molecules to measure nuclear spin-dependent parity violation effects, outlining a detection method based on the internal Josephson effect between molecular states of opposite parity. When applied to molecular condensates, the fine experimental control achieved in atomic bosonic Josephson junctions could provide data on anapole moments and neutral weak couplings.
\end{abstract}

Since the discovery of the electroweak unification, parity violation $(\mathrm{PV})$ has been one of the outstanding problems in atomic and molecular physics. Although the success of the standard model (SM) of elementary particles is extraordinary, the search for new physics is still carried out at high- and medium-energy particle colliders. On the other hand, very sensitive low-energy probes can also be implemented to test SM predictions [1]. As compared to large accelerators, atomic experiments offer a complementary approach to study PV. In particular, nuclear spin-dependent PV effects focus on two causes. One is the electroweak neutral coupling between electron vector- and nucleon axial-vector currents. This can be parametrized by two constants, $\mathrm{C}_{2 u / d}$, describing the electron couplings to up/down quarks. These constants are the most poorly characterized parameters in the SM [2]. Thus, precise measurements of $\mathrm{C}_{2 u / d}$ are potentially sensitive to new physics beyond the SM [3]. Another cause of spin-dependent PV is the weak nucleon-nucleon interaction which gives place to the anapole moment [4, 5]. This parity violating magnetic moment, which results from the chirality acquired by the nucleon current [6], couples to the spin of the electron. As claimed by Haxton and Wieman [7], the most practical strategy for studying the effects of $Z^{0}$ exchange between hadrons is the investigation of the parity-violating nucleon-nucleon interaction. One of its signatures is the resulting anapole moment, which has only been measured in ${ }^{133} \mathrm{Cs}[8]$. These high precision measurements allow for a better determination of the meson-nucleon parity violating interaction constants, with standard reference values given by the Desplanques, Donohue and Holstein (DDH) best values [9]. As there are large uncertainties in these possible values (the DDH reasonable ranges), it is therefore important to determine the weak coupling constants experimentally.

These and other important tests of the SM could be performed by using the ultra-high resolution which is potentially available in cold molecule experiments. In particular, it is known that the internal structure and specific properties of diatomic molecules can enhance the violation of some discrete symmetries, as compared to their atom counterparts [10]. In addition, heteronuclear molecules exhibit dipole-dipole interactions that make them attractive candidates for use in quantum simulations of condensed-matter systems [11] and in quantum computation [12]. Thus, experiments involving ultracold heteronuclear diatomic molecules will be valuable to a large portion of the physics community. The fact that recently both homonuclear [13] and heteronuclear [14, 15] ultracold molecules have been produced in the rovibrational ground state, suggests that the type of experiments envisaged here may not lie too far in the future.

The total nuclear spin-dependent parity violation effect is given by the Hamiltonian [1]

$$
H_{\mathrm{pv}}=\frac{G_{F}}{\sqrt{2}} \kappa \frac{K}{I(I+1)} \boldsymbol{\alpha} \cdot \mathbf{I} \rho(r),
$$

where $G_{F}$ is the Fermi weak constant, $\mathbf{I}$ is the nuclear spin, $K=(I+1 / 2)(-1)^{I+1 / 2-l}, l$ is the orbital angular momentum of the unpaired nucleon, $\boldsymbol{\alpha}$ is the vector whose components are the Dirac matrices acting on the electron spinor, and $\rho(r)$ is the nuclear density. The $\kappa$ term is

$$
\kappa=\kappa_{a}-\frac{K-1 / 2}{K} \kappa_{2}+\frac{I(I+1)}{K} \kappa_{Q},
$$

where $\kappa_{a}$ stands for the anapolar term, $\kappa_{2}$ is due to the electroweak neutral coupling between electron vectorand nucleon axial-vector currents and $\kappa_{Q}$ represents the interaction due the nuclear weak charge perturbed by the hyperfine interaction 1]. We note that the nuclear anapole moment is the dominant source of $\mathrm{PV}$ in atoms with atomic number $A \gtrsim 20$ [7] since it increases as $\kappa_{a} \sim g A^{2 / 3}$, where $g$ is the strength of the weak interaction between the unpaired nucleon and the nuclear core (the $\kappa_{Q}$ term also increases as $A^{2 / 3}$ but the numerical coefficient is very small). Let us recall that there have been proposals for measuring the anapole moment in the ground state of heavy alkali-metal atoms [16]. Electroweak calculations show that, for a heavy nucleus such as, e.g., $\mathrm{Rb}, \kappa_{a} / \kappa_{Q}=20$ and $\kappa_{a} / \kappa_{2}=5$ [17].

Concerning molecules, we note that the levels of opposite parity are four to five orders of magnitude closer in diatomic molecules than in atoms, which results in stronger mixing of those levels by PV [10]. The fact that the near-degeneracy of a pair of opposite parity levels enhances the level mixing caused by the weak interaction can be exploited for precision measurements, as shown by the Berkeley group with atomic dysprosium [18]. We note that this enhancement mechanism 
does not work for homonuclear diatomic molecules [19]. Moreover, this energy interval between opposite parity levels can be made even smaller by applying an external magnetic field. In addition, and contrary to the case of anomalously close levels of opposite parity in rare-earthmetal atoms, the natural linewidths of all the levels in the electronic ground state of a molecule are negligible [20]. Most often, the ground state of a molecule which has an odd number of electrons is either the ${ }^{2} \Sigma$ or the ${ }^{2} \Pi_{1 / 2}$ state. However, since the rotational structure of the lower energy levels is simpler in the former case, we will concentrate on ${ }^{2} \Sigma$ diatomics, which are the molecular equivalent of alkali-metal atoms.

Almost all the molecules that have ${ }^{2} \Sigma$ as their ground state pertain to the Hund's $b$ coupling scheme. Following Ref. [20], L (electronic orbital angular momentum) is coupled to $\mathbf{R}$ (rotational angular momentum of the nuclei) to form $\mathbf{N}$, and $\mathbf{N}$ is coupled to $\mathbf{S}$ (electronic spin) to form $\mathbf{J}$. Their corresponding projections on the internuclear axis, $\mathbf{n}$, are, respectively, $m_{L}, m_{N}, m_{S}$ and $m_{J}$. Including nuclear hyperfine interactions by the coupling $\mathbf{J}+\mathbf{I}=\mathbf{F}$, one obtains the spin-rotation-hyperfine Hamiltonian:

$$
H_{\mathrm{srh}}=B \mathbf{N}^{2}+\gamma \mathbf{N} \cdot \mathbf{S}+b \mathbf{I} \cdot \mathbf{S}+c(\mathbf{I} \cdot \mathbf{n})(\mathbf{S} \cdot \mathbf{n}),
$$

where $\gamma$ is the spin-rotation constant and $b, c$ are hyperfine constants.

In most cases of interest, $B \gg \gamma, b, c$, so $N$ is a good quantum number with eigenstates of energy $E_{N} \simeq$ $B N(N+1)$ and parity $P=(-1)^{N}$. Since one can use a magnetic field to Zeeman shift consecutive states of opposite parity $(N=0,1)$, and the magnetic field necessary to overcome the rotational energy is large enough (about $1 \mathrm{~T}$ when the rotational constant is $B \sim 1 \mathrm{GHz}$ ), one can work in the decoupled basis $\left|N, m_{N}\right\rangle\left|S, m_{S}\right\rangle\left|I, m_{I}\right\rangle$.

Within the subspace of rotational-hyperfine levels, the parity violating Hamiltonian (11) can be written effectively [10]

$$
H_{\mathrm{pv}}=W_{p} \kappa(\mathbf{n} \times \mathbf{S}) \cdot \mathbf{I},
$$

where $W_{p}$ can be calculated using semiempirical methods [21].

The opposite-parity levels

$$
\begin{aligned}
|A\rangle & \equiv|0,0\rangle\left|1 / 2, m_{S}\right\rangle\left|I, m_{I}\right\rangle \\
|B\rangle & \equiv\left|1, m_{N}^{\prime}\right\rangle\left|1 / 2,-m_{S}\right\rangle\left|I, m_{I}^{\prime}\right\rangle
\end{aligned}
$$

can be mixed by $H_{\mathrm{pv}}$ when the quantum number $m_{F}=$ $m_{N}+m_{S}+m_{I}$ is identical for both states $|A\rangle$ and $|B\rangle$ [22]. Thus, once $W_{p}$ has been calculated [21] together with the matrix elements of the operator $(\mathbf{n} \times \mathbf{S}) \cdot \mathbf{I}$ between opposite-parity states, we seek to determine $\kappa$ by measuring

$$
i W \equiv \kappa W_{p}\langle A|(\mathbf{n} \times \mathbf{S}) \cdot \mathbf{I}| B\rangle .
$$

Importantly, time reversal invariance ensures that $i W$ is purely imaginary. We note that this scheme is currently being designed to measure $i W$ using a Stark-interference technique [23].

Here we focus on the effect of $H_{\mathrm{pv}}$ in an internal bosonic Josephson junction (BJJ) between two rotational states in a gas of Bose condensed diatomic molecules, achievable with foreseeable advances in molecular laser cooling. In this case, PV effects can be manifested in the population difference and in the relative phase between the two modes, which can be directly measured both in the Rabi and Josephson regimes [24]. The description of the Gross-Pitaevskii (GP) dynamics reduces, at low energies, to a nonlinear two-mode equation for the time-dependent amplitudes, $\psi_{i}(t)=\sqrt{N_{i}(t)} e^{i \theta_{i}}$ $(i=A, B)$, where $N_{i}$ and $\theta_{i}$ are, respectively, the condensate molecule number and phase in state $i$. When the two condensates are coherently linked, we write the wave function as

$$
|\Psi(t)\rangle=\psi_{A}(t)|A\rangle+\psi_{B}(t)|B\rangle,
$$

where $N_{A}(t)+N_{B}(t)=N_{T}$.

Including $H_{\mathrm{pv}}$, the amplitudes and phases obey the GP equation

$$
i \frac{\partial}{\partial t}\left[\begin{array}{l}
\psi_{A} \\
\psi_{B}
\end{array}\right]=\left[\begin{array}{cc}
E_{A} & -\Omega / 2+i W \\
-\Omega / 2-i W & E_{B}
\end{array}\right]\left[\begin{array}{l}
\psi_{A} \\
\psi_{B}
\end{array}\right]
$$

where $E_{i}$ is the effective energy (which includes the meanfield contribution), and $\Omega$ is the highly tunable Rabi frequency connecting the two modes [25 27].

Defining the fractional population imbalance, $z(t) \equiv$ $\left[N_{A}(t)-N_{B}(t)\right] / N_{T}$, the relative phase, $\phi(t) \equiv \theta_{A}(t)-$ $\theta_{B}(t)$, and rescaling to a dimensionless time $\Omega t \rightarrow t$, we obtain

$$
\begin{aligned}
\dot{z} & =-\sqrt{1-z^{2}}(\sin \phi+w \cos \phi) \\
\dot{\phi} & =\Lambda z+\varepsilon \\
& +\frac{z}{\sqrt{1-z^{2}}}(\cos \phi-w \sin \phi),
\end{aligned}
$$

where $\varepsilon$ is the effective detuning, $\Lambda \equiv U N_{T} / \Omega, w \equiv$ $2 W / \Omega$ and $U$ encompasses the various interactions. We note that these equations represent a generalization of the well known pendulum equations that describe the classical dynamics of two weakly coupled Bose-Einstein condensates 25, 26]. They clearly show the PV effect in a molecular BJJ, with the parity violating contribution $W$ competing with the usual coupling matrix element $\Omega / 2$. We conclude that electroweak effects will have measurable consequences on the macroscopic dynamics of the condensate.

Equations (9) derive from the Hamiltonian

$$
H=\frac{\Lambda z^{2}}{2}+\varepsilon z-\sqrt{1-z^{2}}(\cos \phi-w \sin \phi),
$$

which describes a parity-violating non-rigid pendulum, where the parity operation in this representation changes $\phi \rightarrow-\phi$. Within this mechanical analog, PV is reflected in the shifted equilibrium position of the pendulum, which now is $\phi_{w}=\arctan w$ due to the $\sin \phi$ term in (10). 
For $\varepsilon=0$ and negligible interactions $(\Lambda=0)$, the oscillations have the modified Rabi frequency

$$
\bar{\Omega}=\Omega \sqrt{1+w^{2}} .
$$

In this simple case, the population imbalance evolves as

$$
z(t)=\sqrt{\frac{A_{1}}{1+w^{2}}} \sin \left(\bar{\Omega} t+A_{2}\right),
$$

where $A_{1}=1+w^{2}-H^{2}$ and $A_{2}=$ $\sin ^{-1}\left[z_{0} \sqrt{\left(1+w^{2}\right) / A_{1}}\right]$, with $z_{0} \equiv z(0)$. The evolution of $\phi(t)$ is also very sensitive to $i W$ but cannot be written in closed form.

Deviation from the standard non-rigid pendulum dynamics is shown in the time dynamics of both the population imbalance and phase difference (Fig. 1).
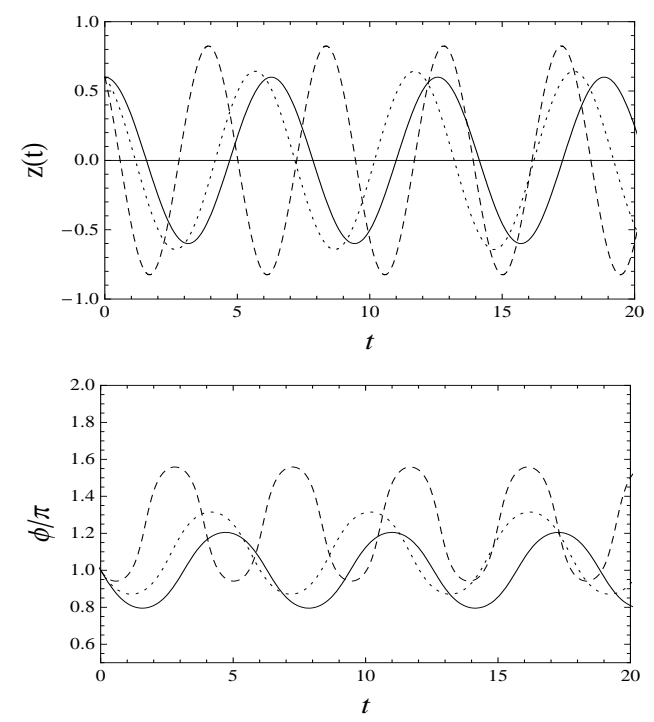

Figure 1. Time evolution of the population imbalance and phase difference for $\Lambda=0$ and $w=0$ (solid), 0.3 (dotted) and 1.0 (dashed). We have taken $z_{0}=0.6$ and $\phi_{0}=\pi$.

In the presence of interactions [28], the harmonic limit $\left(|z|,\left|\phi-\phi_{w}\right| \ll 1\right)$ displays sinusoidal oscillations of $z(t)$ with a modified Josephson-Rabi frequency

$$
\bar{\omega}_{\mathrm{JR}}=\bar{\Omega}(1+\bar{\Lambda})^{1 / 2},
$$

where $\bar{\Lambda}=\Lambda / \sqrt{1+w^{2}}$ is the PV-renormalized interaction parameter. The linearized Eqs. (9) read

$$
\begin{aligned}
& \ddot{z}+\bar{\omega}_{\mathrm{JR}}^{2} z=0 \\
& \ddot{\phi}+\bar{\omega}_{\mathrm{JR}}^{2}\left(\phi-\phi_{w}\right)=0 .
\end{aligned}
$$

We conclude that in the harmonic limit, PV effects change the Josephson-Rabi frequency. In the noninteracting limit, the physics is formally identical to that of a non-degenerate gas of molecules, if the external fields are uniform. In the presence of inhomogeneities, an important advantage of the condensate is that it permits an identical response from all molecules, which should improve the accuracy of the experiment. Following Ref. [29], we estimate that the condensate response to a spatially varying Rabi frequency in the $z$ axis is rigid when $E_{1}-E_{0}>\left|\Omega^{\prime}\right|\langle 1|z| 2\rangle$, where $\Omega^{\prime}$ stands for the gradient of the Rabi frequency, and 1 and 2 refer to the two lowest states of the trap. Taking typical values for trapping frequencies of $2 \pi \times 5 \mathrm{~Hz}$ and trap sizes of about 25 $\mu \mathrm{m}$, we obtain that the condensate response is rigid when $\left|\Omega^{\prime}\right|<2 \pi \times 0.1 \mathrm{~Hz} \mu \mathrm{m}^{-1}$.

In the strongly nonlinear regime, macroscopic quantum self trapping (MQST) is also present [25]. In fact, the value $z_{0}=0$ is inaccesible at any time if

$$
\Lambda>\bar{\Lambda}_{c}=\Lambda_{c}+\frac{w}{z_{0}^{2}} \sqrt{1-z_{0}^{2}} \sin \phi_{0},
$$

where $\phi_{0} \equiv \phi(0)$ and $\Lambda_{c}=\frac{2}{z_{0}^{2}}\left(\sqrt{1-z_{0}^{2}} \cos \phi_{0}+1\right)$ is the critical parameter for MQST in absence of PV effects. In Fig. 2 we show, for $\Lambda=\Lambda_{c}=1.11$, the comparison of parity-even and parity-odd effects in the Fourier transform of the population imbalance, noting that the PV effects are easily distinguishable from their parity conserving counterpart. We notice that the harmonic content of $z(t)$ is quite sensitive to $i W$ for large $\Lambda$. In the inset we explicitly show the effect of PV in the time evolution of the population imbalance. We note that, due to the difference between $\bar{\Lambda}_{c}$ and $\Lambda_{c}$, the onset of MQST depends on the value of $w$.

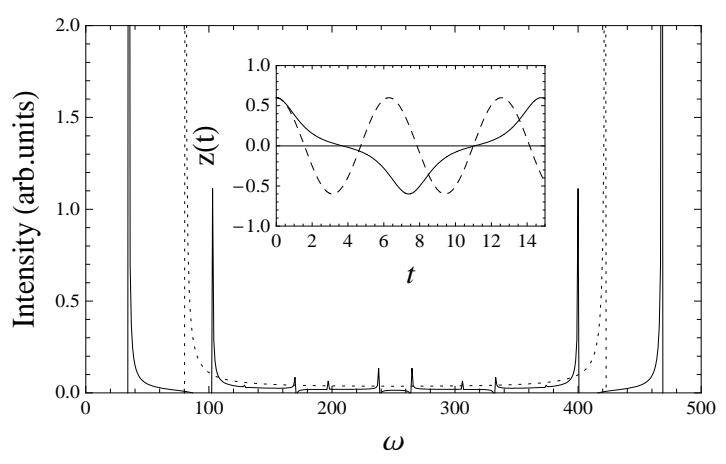

Figure 2. Time evolution of the population imbalance (inset) and its Fourier transform for $\Lambda=\Lambda_{c}=1.11$ and $w=0$ (solid) and 0.2 (dotted).

Once an appropriate control over the internal states of diatomic molecules is achieved, one also needs a precise measurement of the conjugate variables $z, \phi[24$ to measure PV effects in a molecular BJJ setup. Hereafter we focus on the molecule ${ }^{87} \mathrm{Rb}^{A} \mathrm{Yb}$ (with even $A$ ). Ytterbium is so far one of the few elements besides the alkali metals that can be trapped and condensed in optical traps [30]. In addition, as $\mathrm{Yb}$ is a closed $s$-shell atom with zero nuclear spin, the only active electron in the molecule is the $\mathrm{Rb} s$-electron. Thus, from the point of view of the electroweak interaction, the $\mathrm{Yb}$ atom plays a passive role. 
First we estimate the value of $\kappa .{ }^{87} \mathrm{Rb}$ has $I=3 / 2$ with an unpaired proton in the $p$ state. Thus, $\kappa=$ $\kappa_{a}+\frac{5}{4} \kappa_{2}-\frac{15}{8} \kappa_{Q}$. To get an insight on the order of magnitude expected for the parity violating effect, we consider $\kappa \simeq \kappa_{a}$ [7, 17]. Taking $g_{p} \simeq 5$ [7], we get $100 \kappa_{a} \simeq$ 27. Given the proportionality between matrix elements of different weak interactions, we estimate the value of $W_{p}$ using previous molecular electronic structure calculations of the electron electric dipole moment of $\mathrm{RbYb}$ [31], getting $W_{p} \simeq 36 \mathrm{~Hz}$ (for comparison, we note that $\mathrm{RaF}$ has one of the largest values for $W_{p}$ predicted so far, which is in the range of $\mathrm{kHz}$ [32]). Thus, we conclude that the extra coupling that has to be accounted for in a RbYb-BJJ is, including only the anapolar term, $W \simeq 4 \mathrm{~Hz}$. If we include also the contribution of $\kappa_{2} \simeq 0.05$ and $\kappa_{Q} \simeq 0.01$ [17], we obtain $W \simeq 5 \mathrm{~Hz}$.

The Rabi frequency $\Omega$ is a highly tunable parameter because of its linear dependence on the applied dc electric field. We note that $\mathrm{RbYb}$ has a typical value for the electric dipole matrix element of $1 \mathrm{kHz} \mathrm{cm} \mathrm{V}^{-1}$. Thus, for instance, a value $\Omega \sim 100 \mathrm{~Hz}$ can be obtained using an electric field $\sim 0.1 \mathrm{~V} \mathrm{~cm}^{-1}$. Due to the small value of this matrix element, dipole-dipole interactions are negligible compared to both $\Omega$ and $W$. In addition, we note that Zeeman degeneracy can be controlled up to $10^{-2} \mathrm{~Hz}$ for $\mathrm{RbYb}$ due to the high level of magnetic field control (few tens of $\mu \mathrm{G}$ ) recently achieved in a cold atom experiment [33].

The curves $z(t)$ [Eq. (12)] and $\phi(t)$ (which lacks a closed form but can be calculated numerically with arbitrary accuracy) depend on $w=2 W / \Omega$ through their frequency and amplitude. If we focus on the Rabi regime, where interactions are negligible, and assume that we estimate the value of $w$ only from the measurement of the oscillation frequency, the resolution obtained for $W \simeq 5$ $\mathrm{Hz}$ is known to be $\Delta W \sim f /\left(\tau \sqrt{N_{T}}\right)$, where $\tau$ is the coherence time and $f$ is numerical factor taking into account recent experimental capabilities in the absorption imaging of ultracold molecules. We take $N_{T} \simeq 4 \cdot 10^{4}$ as the total number of condensed molecules and assume $\tau \sim$
$100 \mathrm{~ms}$. Following Ref. [34] we take $f=20$ and get that the effect of $H_{\mathrm{pv}}$ could be experimentally detected within a relative precision of $\sim 20 \%$. We point out that this improvement in the accuracy of $\Delta W$ is due to the long coherence times of trapped cold molecules, as compared to those of a molecular beam experiment 32]. Concerning this coherence time, an important point is the role of losses. For RbYb we expect a loss time of about 100 ms 35. This time should be larger than both the Rabi and PV times, $\Omega^{-1}$ and $W^{-1}$, for this proposal to be viable. The value of $\Omega$ is not a problem since it is highly tunable (or even zero). For RbYb, taking $W \simeq 5 \mathrm{~Hz}$, this condition can hardly be satisfied. However, we note that current estimates of $W_{p}$ are still rather crude and the real situation might turn out to be more favourable. On the other hand, molecules such $\mathrm{RaF}, \mathrm{HgH}$ or $\mathrm{HgF}$ are known to have much larger values of $W_{p}$ [32].

Understanding the final sensitivity that could be reached by using a molecular BJJ requires a careful study of systematic effects. As noted in Refs. [18, 23], care has to be taken of stray electric fields that could mimic the $\mathrm{PV}$ effect. Due to the small value of the electric dipole matrix elements, it suffices to control stray fields within a feasible accuracy of $0.1 \mathrm{~V} \mathrm{~cm}^{-1}$. Thermal effects can be neglected if the temperature is smaller than a typical rotational level splitting $(\sim \mathrm{mK})$. However, temperature can be bigger than $W$ and $\Omega$, thanks to the fine control of the initial state.

We finally note that in the proposed setup a large number of curves depend on a few parameters. This intrinsic redundancy will permit not only a consistency check of the underlying physics but a precise measurement of the anapole moment in a given molecule. The eventual measurement of this moment in a variety of molecules may ultimately reveal the strength of the poorly known electron couplings to up and down quarks.

We acknowledge useful discussions with A. J. Leggett, I. Zapata and A. Dorta-Urra. This work has been supported by MICINN (Spain) through Grants No. FIS2007-65723, No, FIS2010-21372, and No. CTQ200802578/BQU, and the Juan de la Cierva program (P. B.).
[1] J. S. M. Ginges and V. V. Flambaum, Phys. Rep. 397, 63 (2004).

[2] K. Nakamura et al., J. Phys. G. 37, 075021 (2010).

[3] P. Langacker, M. Luo and A. K. Mann, Rev. Mod. Phys. 64, 87 (1992); P. Langacker, Rev. Mod. Phys. 81, 1199 (2009).

[4] Ya. B. Zeldovich, Sov. Phys. JETP 6, 1184 (1958).

[5] V. V. Flambaum, I. B. Khriplovich and O. P. Suskov, Phys. Lett. B 146, 367 (1984).

[6] The anapole moment classically is defined by $\mathbf{a}=$ $-\pi \int d^{3} r r^{2} \mathbf{J}(\mathbf{r})$, where $\mathbf{J}$ is the electromagnetic current density. See, for example, Ref. [19].

[7] W. C. Haxton and C. E. Wieman, Annu. Rev. Nucl. Part. Sci. 51, 261 (2001).

[8] C. S. Wood, S. C. Bennett, D. Cho, B. P. Masterson, J.
L. Roberts, C. E. Tanner, and C. E. Wieman, Science 275, 1759 (1997).

[9] B. Desplanques, J. F. Donoghue, and B. R. Holstein, Ann. Phys. 124, 449 (1980).

[10] V. V. Flambaum and I. B. Khriplovich, Phys. Lett. A 110, 121 (1985).

[11] L. D. Carr, D. DeMille, R. V. Krems, and J. Yu, New J. Phys. 11, 055049 (2009).

[12] D. DeMille, Phys. Rev. Lett 88, 067901 (2002).

[13] J. G. Danzl, M. J. Mark, E. Haller, M. Gustavsson, R. Hart, J. Aldegunde, J. M. Hutson, and H.-C. Nägerl, Nat. Phys 6, 265 (2010).

[14] K. Aikawa, D. Akamatsu, M. Hayashi, K. Oasa, J. Kobayashi, P. Naidon, T. Kishimoto, M. Ueda, and S. Inouye, Phys. Rev. Lett 105, 203001 (2010). 
[15] K.-K. Ni, S. Ospelkaus, D. Wang, G. Quéméner, B. Neyenhuis, M. H. G. de Miranda, J. L. Bohn, J. Ye, and D. S. Jin, Nature, 464, 1324 (2010).

[16] E. Gomez, S. Aubin, G. S. Sprouse, L. A. Orozco, and D. P. DeMille, Phys. Rev. A 75, 033418 (2007); M.-A. Bouchiat, Phys. Rev. Lett. 98, 043003 (2007).

[17] D. Sheng, L. A. Orozco and E. Gomez, J. Phys. B: At. Mol. Opt. Phys. 43, 074004 (2010).

[18] A. T. Nguyen, D. Budker, D. DeMille, and M. Zolotorev, Phys. Rev. A 56, 3453 (1997).

[19] I. B. Khriplovich, Parity Nonconservation in Atomic Phenomena (Gordon and Breach, Philadelphia, 1991).

[20] J. Brown and A. Carrington, Rotational Spectroscopy of Diatomic Molecules (Cambridge University Press, Cambridge, 2003).

[21] M. G. Kozlov, Sov. Phys. JETP. 62, 1114 (1985).

[22] M. G. Kozlov and L. N. Labzowsky, J. Phys. B: At. Mol. Opt. Phys. 28, 1933 (1995).

[23] D. DeMille, S. B. Cahn, D. Murphree, D. A. Rahmlow, and M. G. Kozlov, Phys. Rev. Lett. 100, 023003 (2008).

[24] C. Gross, T. Zibold, E. Nicklas, J. Estéve, and M. K. Oberthaler, Nature (London) 464, 1165 (2010); T. Zibold, E. Nicklas, C. Gross, and M. K. Oberthaler, Phys.
Rev. Lett. 105, 204101 (2010).

[25] A. Smerzi, S. Fantoni, S. Giovanazzi, and S. R. Shenoy, Phys. Rev. Lett 79, 4950 (1997).

[26] I. Zapata, F. Sols and A. J. Leggett, Phys. Rev. A R57, 28 (1998).

[27] J. I. Cirac, M. Lewenstein, K. Molmer, and P. Zoller, Phys. Rev. A 57, 1208 (1998).

[28] We assume $\Lambda \ll N_{T}^{2}$ so that global phase coherence and thus the validity of the GP equation is guaranteed.

[29] J. Williams, R. Walser, J. Cooper, E. A. Cornell, and M. Holland, Phys. Rev. A., 61, 033612 (2000).

[30] Y. Takasu, K. Maki, K. komori, T. Takano, K. Honda, M. Kumakura, T. Yabuzaki, and Y. Takahashi, Phys. Rev. Lett. 91, 040404 (2003).

[31] E. R. Meyer and J. L. Bohn, Phys. Rev. A, 80, 042508 (2009).

[32] T. A. Isaev, S. Hoekstra and R. Berger, Phys. Rev. A 82, 052521 (2010).

[33] A. Smith et al., J. Phys. B: At. Mol. Opt. Phys, 44, 205002 (2011).

[34] D. Wang et al., Phys. Rev. A 81, 061404 (2010).

[35] P. S. Julienne, T. M. Hanna and Z. Idziaszek, Phys. Chem. Chem. Phys., DOI: 10.1039/C1CP21270B (2011). 